\title{
Intrathoracic extramedullary hematopoiesis as a posterior mediastinal tumor: a case report
}

\author{
Elçin Ersöz Köse', Gökay Reyhan¹, Serda Kanbur Metin², Serdar Evman², Çağatay Tezel², İrfan \\ Yalçınkaya $^{2}$ \\ ${ }^{1}$ Department of Thoracic Surgery, University of Health Sciences, Sultan Abdülhamid Han Training and Research Hospital, Istanbul, \\ Turkey \\ ${ }^{2}$ Department of Thoracic Surgery, University of Health Sciences, Süreyyapaşa Chest Disease and Thoracic Surgery Training and \\ Research Hospital, Istanbul, Turkey
}

DOI: $10.18621 /$ eurj.345666

\begin{abstract}
Extramedullary hematopoiesis (EMH) is a rare disorder, defined as the appearance of hematopoietic elements outside the bone marrow or peripheral blood. Usually it is diagnosed incidentally and very rarely it presents as a posterior mediastinal mass clinically mimicking with many other benign or malignant mediastinal tumors. Intrathoracic EMH is a rare condition and only very few case reports have been described in patients with chronic myeloproliferative disorders specially myelofibrosis with myeloid metaplasia and spherocytic anemias. The paravertebral area is an uncommon site for EMH, with the possible exception of sporadically reported intrathoracic manifestations. In this study, a 38-year-old male patient was followed for myeloproliferative disease. Tumor-like lesions at the bilateral posterior mediastinum were detected in the patient who applied to our clinic due to fall. Excision was planned with video- assisted thoracoscopic surgery to differentiate from mediastinal tumors, but the mass unblock was removed with posterolateral thoracotomy because of the appearance of the lesion and large size. Histopathologic diagnosis was reported as extramedullary hematopoiesis. Patient was presented because of its rare location.
\end{abstract}

Keywords: Extramedullary hematopoiesis, posterior mediastinum, myeloproliferative disease

Received: October 22, 2017; Accepted: March 20, 2017; Published Online: March 23, 2018

$\mathrm{E}$ xtramedullary hematopoiesis $(\mathrm{EMH})$ is production of blood cells, other than bone marrow, and is a compensatory mechanism of various hematological diseases such as thalassemia, sickle cell anemia, myelofibrosis, hereditary sferositozis. Thorax is a rare area where extramedullary hematopoiesis is seen. EMH focuses are usually microscopic and in some cases they are in the form of mass-like lesions and often seen in liver, spleen, lymph nodes, but have been reported in almost all organs. Intrathoracic EMH

is usually asymptomatic and rarely requires treatment. Treatment is recommended in case of massive pleural effusion and haemothorax presence and when dyspnea develops in pulmonary parenchymal involvement. As a treatment, these patients can be given low-dose radiation therapy. Surgical excision is required in complications including bleeding in the mass, spinal cord compression or spinal canal invasion. In this article, a rare intrathoracic extramedullary hematopoiesis is presented.

Address for correspondence: Elçin Ersöz Köse, MD., University of Health Sciences, Sultan Abdülhamid Han Training and Research Hospital, Department of Thoracic Surgery, Istanbul, Turkey

E-mail: elcinersoz@hotmail.com 


\section{CASE PRESENTATION}

A 38-year old male patient with no complaints has been followed due to myeloproliferative disorder and chronic hemolytic anemia and he has a history of frequent blood transfusion. The patient was examined upon detection of lesions in bilateral mediastinal areas, distinctly in the left side, on the chest X-ray performed 1 month ago after fall history. In the family history of the patient, who had a history of smoking 20 packs/year, existed splenectomy in his sister due to myeloproliferative disease and lung cancer in his father. There were no abnormalities in his physical examination apart from hepatosplenomegaly and icterus in the scleras. FEV1 values were determined as $3.66-89 \%$ in pulmonary function test. In thorax computed tomography (CT) of the patient, distinct nodular homogeneous soft tissue density areas in the left side of bilateral paravertebral region in the mediastinum were observed. In position emission tomography $(\mathrm{PET}) / \mathrm{CT}$, mediastinal masses showing involvement with a size of $36 \times 49 \times 70 \mathrm{~mm}$ in the left lower lobe posteromediobasal segment neighborhood on T8-T10 vertebra (SUV max: 3), and with a size of $11 \times 23 \mathrm{~mm}$ in the right lower lobe mediobasal segment neighborhood on T8 vertebra (SUV max: 2.7 ) were detected (Figure 1). Initially, low grade lymphoma was considered, operation planned for the biopsy. The patient, for whom preoperative hematology consultation was required, was recommended $20 \mathrm{mg} / \mathrm{kg}$ TDP. At first, we planned the posterior mediastinal mass biopsy with video-assisted thoracoscopy (VATS) (Figure 2). The macroscopic image of the lesion removed us from the lymphoma, and the mass had a size of $7 \times 5 \mathrm{~cm}$, so we decided to

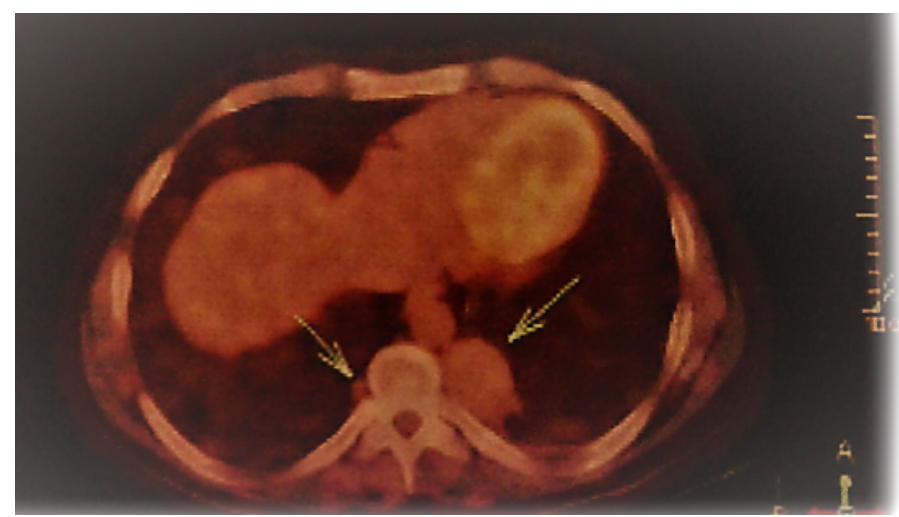

Figure 1. Bilateral posterior mediastinal masses in PET-CT scans

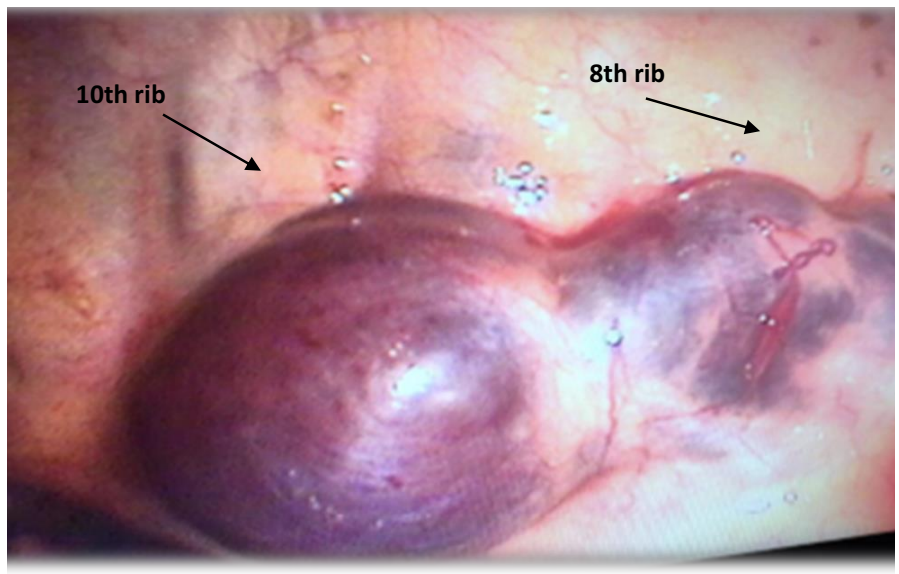

Figure 2. Peroperative appearance of the posterior mediastinal mass at the VATS.

remove all of the mass with thoracotomy incision. The left posterolateral incision was inserted into the thorax from the fifth intercostal space. The mass adhered to posterior wall of the chest, and the advanced adhesions were separated with the dissection. All of the lesion was removed unblock. Postoperative hemogram values were within normal limits. His drain was terminated in the post-operative third day and he was discharged in the postoperative fourth day. The pathology report was initially reported as Hodgkin lymphoma but the final report was finalized as extramedullary hematopoiesis. As hemogram fell to $5 \mathrm{~g} / \mathrm{dl}$ in the postoperative first month follow-up, it was applied to hematology and splenectomy was performed on the outer center. The patient showed no recurrence during the follow-ups

\section{DISCUSSION}

Extramedullary hematopoiesis (EMH) is blood production in various parts of the body as a compensatory mechanism in the course of various blood diseases. EMH usually occurs as a response to bone marrow erythropoiesis fault and it can be seen in myeloproliferative disorders, hemoglobinopathies or bone marrow infiltration [1]. In most cases, it is situated in the posteroinferior mediastinum, and usually accompanied by chronic hemolytic anemia presenting in a male adult predominantly. These intrathoracic neoplasms could be solitary or multiple masses, even unilaterally or bilaterally [2]. Similar to the literature in our case, adult male patient has been 
followed due to myeloproliferative disorder and chronic hemolytic anemia and he has a history of frequent blood transfusion.

Mimicking tumor in the intrathoracic cavity, presence of EMH, especially as posterior mediastinal mass, is very rare. EMH is usually characterized by development of soft tissue masses in the paravertebral area in the thorax. These masses very rarely cause distinct symptoms but may lead to hemothorax and pleural effusion [3]. In our case, the case was asymptomatic and EMH was detected incidentally. Magnetic resonance imaging (MRI) and thorax computed tomography (CT) scans are the most commonly used diagnostic method for EMH. They appear as lobular masses in the paravertebral region [4]. In diagnosis, PET/CT scan may be useful. So far, only a few cases have been reported with an EMH diagnosis, using PET/CT scan [5] and it can be considered as a benign mass with low SUV max values and normal appearance in the tissue. Concomitant presence of underlying hematopoietic disorder suggests EMH diagnosis. In our case, we determined masses in the bilateral paravertebral region at the thorax CT incidentally. The patient underwent $\mathrm{PET} / \mathrm{CT}$ to differentiate between benign and malignant lesion. PET/CT scan showed that the lesion had low SUV max values.

Although fine-needle aspiration biopsies or surgical biopsies support the diagnosis, they are not recommended for those with underlying hematologic diseases due to bleeding risk. In the presence of anemia, preoperative EMH diagnosis can be considered, however EMH was not considered as a potential diagnosis initially in our case because hemogram values were normal in preoperative examinations.

Hematopoietic tissue is particularly radiosensitive and it undergoes shrinkage with radiotherapy; excellent results have been obtained with radiotherapy alone in EMH patients. In EMH patients, surgical excision is required in complications including bleeding in the mass, spinal cord compression or spinal canal invasion [6]. Such an excision not only allows controlling bleeding and/or decompression, but also provides histological diagnosis. In EMH, postoperative radiotherapy may prevent reoccurrence or facilitate complete remission [6]. We planned incisional biopsy because we thought the lymphoma before surgery, but the macroscopic view of the lesion removed us from the lymphoma. At first if we thought EMH, we could plan radiotherapy instead of surgery.

\section{CONCLUSION}

If symmetric posterior mediastinal masses are detected in the patients with hematologic diseases, extramedullary hematopoiesis should be considered in the differential diagnosis. Surgical resection and radiotherapy should be considered as treatment options for EMH.

\section{Informed consent}

Written informed consent was obtained from the patient for the publication of this case report.

\section{Conflict of interest}

The authors declared that there are no potential conflicts of interest with respect to the research, authorship, and/or publication of this article.

\section{REFERENCES}

[1] An J, Weng Y, He J, Li Y, Huang S, Cai S, et al. Intrathoracic extramedullary hematopoiesis presenting as tumor-simulating lesions of the mediastinum in $\alpha$-thalassemia: a case report. Oncol Lett 2015;10:1993-6.

[2] Ng CS, Wan S, Lee TW, Sihoe AD, Wan IY, Arifi AA, et al. Videoassisted thoracic surgery for mediastinal extramedullary hematopoiesis. Ann R Coll Surg Engl 2002;84:161-3.

[3] Özdemir L, Özdemir B, Durkaya S, Topal C, Çalışkan SN, Ersoy A, et al. [A rare cause of posterior mediastinal masses: extramedullary hematopoiesis (two cases)]. Respir Case Rep 2014;3:156-8. [Article in Turkish]

[4] Paydaş S, Sargın Ö, Gönlüşen G. PET CT imaging in extramedullary hematopoiesis and lung cancer surprise in a case with thalassemia intermedia. Turk J Haematol 2011;28:60-2.

[5] Zade A, Purandare N, Rangarajan V, Shah S, Agrawal A, Ashish J, et al. Noninvasive approaches to diagnose intrathoracic extramedullary hematopoiesis: 18F-FLT PET/CT and 99mTc-SC SPECT/CT scintigraphy. Clin Nucl Med 2012;37:788-9.

[6] Yeom SY, Lim JH, Han KN, Kang CH, Park IK, Kim YT. Extramedullary hematopoiesis at the posterior mediastinum in patient with hereditary spherocytosis: A case report. Korean J Thorac Cardiovasc Surg 2013;46:156-8. 